\title{
Charge carrier self-organization in ferroelectromagnetic semiconductors $\mathrm{Eu}_{0.8} \mathrm{Ce}_{0.2} \mathrm{Mn}_{2} \mathrm{O}_{5}$
}

\author{
E.I. Golovenchits, V.A. Sanina, V.G. Zalesskii, and M.P. Scheglov \\ A.F. Ioffe Physical Technical Institute of the RAS, 26 Politekhnicheskaya, St. Petersburg 194021, Russia \\ E-mail: sanina@mail.ioffe.ru
}

Received November 9, 2009

\begin{abstract}
The state with a giant permittivity $\left(\varepsilon^{\prime} \sim 10^{4}\right)$ and ferromagnetism has been observed above $185 \mathrm{~K}$ (including room temperature) in single crystals of diluted semiconductor manganite-ferroelectromagnetic $\mathrm{Eu}_{0.8} \mathrm{Ce}_{0.2} \mathrm{Mn}_{2} \mathrm{O}_{5}$ in the investigations of $\mathrm{x}$-ray diffraction, dielectric and magnetic properties, conductivity. X-ray diffraction study has revealed a layered superstructure along the $c$ axis at room temperature. A model of the state with a giant $\varepsilon^{\prime}$ including as-grown 2D layers with doping impurities, charge carriers, and double-exchange coupled $\mathrm{Mn}^{3+}-\mathrm{Mn}^{4+}$ ion pairs is suggested. At low temperatures these layers form isolated electrically neutral small-size 1D superlattices, in which de Haas van Alphen oscillations were observed. As temperature grows and hopping conductivity increases, the charge carrier self-organization in the crystal cause formation of a layered superstructure consisting of charged layers (with an excess $\mathrm{Mn}^{3+}$ concentration) alternating with dielectric layers of the initial crystal - the ferroelectricity state due to charge ordering. Ferromagnetism results from double exchange between $\mathrm{Mn}^{3+}$ and $\mathrm{Mn}^{4+}$ ions through of charge carriers in the charged layers.
\end{abstract}

PACS: 75.47.Lx Magnetic oxides;

76.50.+g Ferromagnetic, antiferromagnetic, and ferrimagnetic resonances; spin-wave resonance;

77.80.-e Ferroelectricity and antiferroelectricity.

Keywords: multiferroic, ECMO, x-ray diffraction, dielectric and magnetic properties, conductivity.

\section{Introduction}

G.A. Smolensky with colleagues synthesized and researched many new materials, among which ferroelectrics, ferroelectromagnetics (now renamed as multiferroics), and magnetic materials. In the laboratory of Ferroelectricity and Magnetism, which was organized by G.A. Smolensky, in a line with traditions the new materials of the ferroelectromagnetic-semiconductors $\mathrm{R}_{1-x}^{3+} \mathrm{Ce}_{x}^{4+} \mathrm{Mn}_{2} \mathrm{O}_{5}$ ( $\mathrm{R}$ - rare-earth ions, $\mathrm{Y}$ and $\mathrm{Bi}$ ) recently were brought up and researched. The paper is devoted to the description of the results of study one crystal from family of such materials.

Orthorhombic crystals of manganites $\mathrm{RMn}_{2} \mathrm{O}_{5}$ are ferroelectromagnetics in which long-range magnetic and ferroelectric orders are realized at $T<30-40 \mathrm{~K}$. A strong coupling between magnetic and ferroelectric states resulting from nearly equal temperatures of magnetic and ferroelectric orderings attracts a considerable attention of researchers to these materials (see [1-4] and references therein). Ferroelectromagnetics with similar ordering temperatures offer a unique possibility to manipulate the magnetic and polar states by applying electric and magnetic fields, respectively, which makes them attractive can- didates for different applications, especially when coupled magnetic and polar states exist at room temperature.

In an attempt to prepare the material with magnetic and ferroelectric ordering temperatures high enough but still close to each other, we grew diluted semiconductor $\mathrm{Eu}_{0.8} \mathrm{Ce}_{0.2} \mathrm{Mn}_{2} \mathrm{O}_{5}$ (ECMO) single crystals. A strong coupling between magnetic and polar states in ferroelectromagnetics-semiconductors can arise at sufficiently high temperatures if charge carriers participate in formation of both the magnetic (through double exchange interaction $[5,6])$ and ferroelectric (through charge ordering [7]) longrange orders, which was demonstrated in our investigations of multiferroic-semiconductor $\mathrm{Tb}_{0.95} \mathrm{Bi}_{0.05} \mathrm{MnO}_{3}$ [8-11].

This paper reports on complex studies of x-ray diffraction, magnetic and dielectric properties of single crystals of ECMO in which the state with a giant $\left(\varepsilon^{\prime} \sim 10^{4}\right)$ permittivity has been observed above $185 \mathrm{~K}$. It has been found that the nature of the high-temperature state in ECMO differs from that of the low-temperature $(T \leq 40 \mathrm{~K})$ improper ferroelectricity in the initial EuMn ${ }_{2} \mathrm{O}_{5}$ (EMO) crystals induced by a complicated magnetic structure [1-4]. Electron doping with $\mathrm{Ce}^{4+}$ ions that substitute $\mathrm{Eu}^{3+}$ ions produces charge carriers, 
and the ratio between $\mathrm{Mn}^{3+}$ and $\mathrm{Mn}^{4+}$ concentrations changes during crystal growth. As a result, the character of charge ordering typical of initial non-diluted crystals with equal $\mathrm{Mn}^{3+}$ and $\mathrm{Mn}^{4+}$ concentrations changes.

The state of phase separation into conductive ferromagnetic and dielectric antiferromagnetic domains typically observed in magnetic semiconductor $\mathrm{La}_{1-x} \mathrm{~A}_{x} \mathrm{MnO}_{3}$ $(\mathrm{A}=\mathrm{Ca}, \mathrm{Ba}, \mathrm{Sr})[5,6]$ occurs in magnetic semiconductor ferroelectromagnetics ECMO as well. However, the symmetry of this phase separation in our crystal is different. Manganese $\mathrm{Mn}^{3+}$ and $\mathrm{Mn}^{4+}$ ions in EMO are arranged in neighboring atomic layers normal to the $c$ axis. The layered arrangement of manganese ions is preserved at crystal doping, but the $\mathrm{Mn}^{3+}$ concentration becomes higher than that of $\mathrm{Mn}^{4+}$. Phase separation and charge selforganization result in new ferroelectric and magnetic states in ECMO at all temperatures.

At low temperatures only a small volume of ECMO crystal is modified by doping. This small volume is comprised of as-grown 2D semiconductor superlattices normal to the $c$ crystal axis and containing doping impurities, charge carriers, and ferromagnetic $\mathrm{Mn}^{3+}-\mathrm{Mn}^{4+}$ ion pairs. These superlattices appreciably change the processes of magnetization and dielectric relaxation in doped crystals as compared with initial crystals.

At high temperatures $(>185 \mathrm{~K})$, a layered superstructure is formed in the doped crystal. A periodic alternation of charged and dielectric layers gives rise to the state of charge-orderinginduced ferroelecticity with a giant permittivity. It has been found that an application of a magnetic field along the $c$ axis manipulates by the ECMO magnetic and polar states. Note that earlier a highly polarized state that changed on application of a magnetic field in an irreversible manner was observed in a diluted multiferroic $\mathrm{TbBiMnO}_{3}[9,11]$.

\section{Experimental techniques and the object of study}

Single crystals of ECMO were grown by the flux method using the same technology as for the initial EMO crystals [12]. The crystal symmetry and composition were determined by x-ray phase analysis and the x-ray fluorescent method, respectively. In additional intensity distribution of Bragg reflections for EMO and ECMO crystals were carried out with a high-sensitivity three-crystal x-ray diffractometer. The ECMO symmetry at room temperature did not differ from that of the initial EMO crystals (space group Pbam). The unit cell volume varied only slightly at doping. Lattice constants of ECMO were: $a=7.44( \pm 0.01) \AA, b=8.42( \pm 0.01) \AA, c=$ $=5.71( \pm 0.01) \AA$, while for EMO they were: $a=7.4003 \AA$, $b=8.5663 \AA, c=5.6972 \AA$.

Ions in $\mathrm{RMn}_{2} \mathrm{O}_{5}$ crystals lie in the layers normal to the crystal $c$ axis [2]. $\mathrm{R}^{3+}$ ions are in the $z=0$ layers and have surrounding $\left(\mathrm{RO}_{8}\right) . \mathrm{Mn}^{4+}$ ions $\left(t_{2 g}^{3} e_{g}^{0}\right)$ are in the $z=0.25 \mathrm{c}$ and $1-z=0.75 \mathrm{c}$ layers and have octahedral surrounding $\left(\mathrm{MnO}_{6}\right)$. The octahedra with common apical oxygen ions are elongated along the $c$ axis and form ribbons. $\mathrm{Mn}^{3+}$ ions $\left(t_{2 g}^{3} e_{g}^{1}\right)$ lie in the $z=0.5 \mathrm{c}$ layers in pyramids formed by five oxygen ions $\left(\mathrm{MnO}_{5}\right)$.

A variable valence of $\mathrm{Ce}^{3.75+}$ ions is typically realized in cerium-containing crystals. The $\mathrm{Ce}^{4+}$ ion radius is smaller than that of $\mathrm{Ce}^{3+}(1.11 \AA$ and $1.28 \AA$, respectively, at coordination number $Z=8$ [13]). The $\mathrm{Eu}^{3+}$ ion radius is $1.21 \AA$ [13]. In view of a smaller size of $\mathrm{Ce}^{4+}$ ions and also the data of Ref. 14, it can be supposed that the main doping ion in the crystals studied is $\mathrm{Ce}^{4+}$. Doping results in a free electron $\left(\mathrm{R}^{3+}=\mathrm{Ce}^{4+}+e\right)$. Free electrons in ECMO propagate in the subsystem of manganese ions, which is typical of manganites $[5,6]$. The nearest neighbors of the layers with $\mathrm{R}^{3+}$ and $\mathrm{Ce}^{4+}$ ions are those with $\mathrm{Mn}^{4+}$ ions. The free electrons in the $\mathrm{Mn}^{4+}$ layer provide recharging $\mathrm{Mn}^{4+}+e \leftrightarrow \mathrm{Mn}^{3+}$. Thus, doping impurities and charge carriers in ECMO are located in neighboring atomic layers perpendicular to the crystal $c$ axis.

Dielectric measurements of capacity and conductivity were carried out with a Good Will LCR-819 impedance meter in the temperature interval 5-350 K and frequency range $250 \mathrm{~Hz}-100 \mathrm{kHz}$. The dc-current conductivity $\sigma_{d c}$ and the ac-current conductivity $\sigma_{a c}$ were measured by a four-contact and capacity methods, respectively. Dielectric spectra were recorded with a Newtons 4th Ltd PSM 1735 device at frequencies $100 \mathrm{~Hz}-5 \mathrm{MHz}$ in the temperature range $120-300 \mathrm{~K}$. Capacitors in the form of $\sim 0.5 \mathrm{~mm}$ thick plates cut from the single crystals perpendicular to the principal crystal axes were used. Contacts were made by gold evaporation. The magnetic moment of ECMO was measured with a vibration magnetometer in magnetic fields of up to $35 \mathrm{kOe}$ oriented along the $a$ and $c$ crystal axes.

\section{X-ray diffraction studies}

Diffraction studies were performed for several samples of ECMO and EMO at room temperature. The angular intensity distribution of $(004)_{\mathrm{CuKa1}}$ Bragg reflections was detected in the 3-crystal regime with the $\theta-2 \theta$ scan having a resolution of $10^{\prime \prime}$. The rocking curve half-width $\omega_{\theta}$ for one of the Bragg peaks was measured in the $\theta$-scan regime, and the lattice constant $d$ was inferred from the exact Bragg angle $\theta_{B}$ of this peak. As a monochromator and an analyzer, germanium crystals in the (004) reflection were used, which allowed conditions of nearly dispersion-free high-resolution survey geometry to be realized.

Figures $1, a$ and $b$ show diffraction curves for ECMO and EMO, respectively. The curve for ECMO exhibits a set of diffraction maxima separated into two pronounced regions. Each region has 5-6 peak pulsations, one of which is dominating in intensity. Both regions have similar widths and number of maxima. Analysis of the diffraction curve has led to the conclusion that the maxima have different natures. There are layer peaks whose positions are determined by parameter $d$ and which characterize the layered crystal 


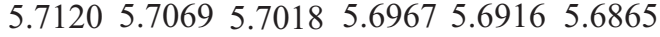
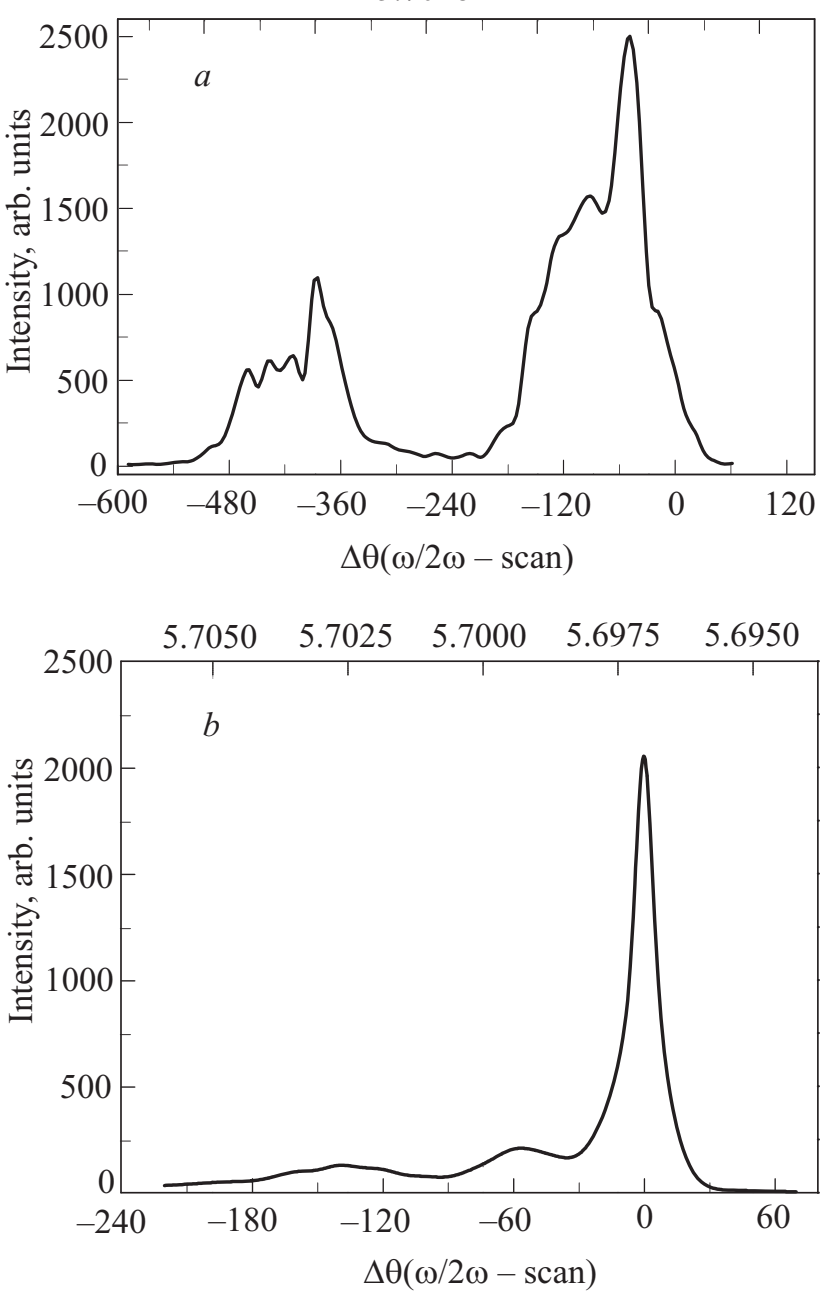

Fig. 1. Angular distribution of Bragg $(004)_{\mathrm{CuKal}}$ reflection intensity for ECMO $(a)$ and EMO $(b)$ crystals. The upper axes show the lattice parameters $d$ along the $c$ axes.

structure. The distances between the maxima $(\Delta \theta)$ of these peaks give information on the lattice mismatch in the layers. In addition, the diffraction curve exhibits periodic intensity oscillations with the $d$-independent period. All five intensity maxima in the left-hand region of the diffraction curve are layer peaks, while only two maxima in the right-hand region are layer peaks, the remaining peaks being oscillatory. The oscillation period $\Delta \theta$ is determined by the structural layer thicknesses $\left(t=\lambda /\left(\Delta \theta 2 \cos \theta_{B}\right)\right.$, where $\lambda$ is the wavelength). In the right-hand region of the curve $\Delta \theta=30^{\prime \prime}$, and the layer thickness is $\sim 750 \AA$. In the left-hand region the layers are thinner. The half-width of the rocking curve $\left(\omega_{\theta}\right)$ for the layer with the highest peak in the right-hand region is $20^{\prime \prime}$, which points to a high structural layer quality.

Figure 1,b shows the diffraction curve for EMO. It consists of one intense narrow peak pointing to a highquality crystal structure in the bulk $\left(\omega_{\theta}=12^{\prime \prime}\right)$ and two low-intensity broad peaks with oscillations pointing to the presence of a two-layer deformed area at the crystal surface. The layer thickness corresponding to the oscillation period $\left(\Delta \theta=23^{\prime \prime}\right)$ is $t \sim 900 \AA$.

Thus, room-temperature $\mathrm{x}$-ray studies of the undoped EMO and doped ECMO crystals have shown that the former has a single-crystal structure with a weak layer inhomogeneity in the near-surface area and the latter has a layered superstructure.

\section{Low temperatures}

\subsection{Dielectric properties}

Figure 2 presents dielectric properties of ECMO at low temperatures. As can be seen from the inset to Fig. 2,c, the temperature dependence of the real part of permittivity $\varepsilon^{\prime}$ of undoped EMO exhibits maxima in the vicinity of 20 and $35 \mathrm{~K}$ when the ac electric field $(\mathbf{E})$ is along the $\mathbf{a}$ and $\mathbf{b}$ axes. At $\mathbf{E} \| \mathbf{c}$ there are no anomalies. A sharp maximum at $T \approx 35 \mathrm{~K}$ corresponds to a ferroelectric phase transition $[15,16]$. Note that the $\varepsilon^{\prime}$ magnitudes for the initial and doped crystals at low temperatures are nearly equal.

No distinct $\varepsilon^{\prime}$ maxima are observed for the doped ECMO. However, for $\mathbf{E} \| \mathbf{b}$ (Fig. 2,b) there is a broad $\varepsilon^{\prime}$ maximum without frequency dispersion at $T \sim 30 \mathrm{~K}$ attributable to the ferroelectric phase transition similar to that observed in EMO at almost the same temperature. This indicates that the major doped crystal volume is occupied by the initial EMO and only a small volume is modified by doping.

At $\mathbf{E} \| \mathbf{a}$ (Fig. 2,a) there is no even a diffuse $\varepsilon^{\prime}$ maximum at $T \approx 30 \mathrm{~K}$. However, relaxation processes with frequency dispersion are observed above $20 \mathrm{~K}$. They manifest themselves as $\varepsilon^{\prime}$ steps and dielectric dissipation maxima $\operatorname{tg} \delta\left(\operatorname{tg} \delta=\varepsilon^{\prime \prime} / \varepsilon^{\prime}, \quad \varepsilon^{\prime \prime}\right.$ is the imaginary part of permittivity).

At $\mathbf{E} \| \mathbf{c}$ (Fig. 2,c), the $\varepsilon^{\prime}(T)$ dependences are nearly linear in a wide temperature range below $100 \mathrm{~K}$ with a change in slope at $T \approx 40 \mathrm{~K}$. The permittivity $\varepsilon^{\prime}$ is frequency-dependent: the higher the frequency, the lower the $\varepsilon^{\prime}$ magnitude. Note that $\varepsilon^{\prime}$ has similar frequency dependences for all $\mathbf{E}$ orientations.

It is natural to attribute the differences in dielectric properties of the initial and doped crystals to the effect of doping. As noted above, at low temperatures a small doped crystal volume is occupied by the as-grown $2 \mathrm{D}$ layers normal to the c axis. These layers differ in the number of atomic layers, concentrations of doping impurities and charge carriers, and barriers at the boundaries and can be regarded as 1D semiconductor superlattices formed in the single crystal bulk. At low temperatures the superlattices interact with each other only slightly, and the number of charge carriers in them is governed by the electroneutrality condition. 

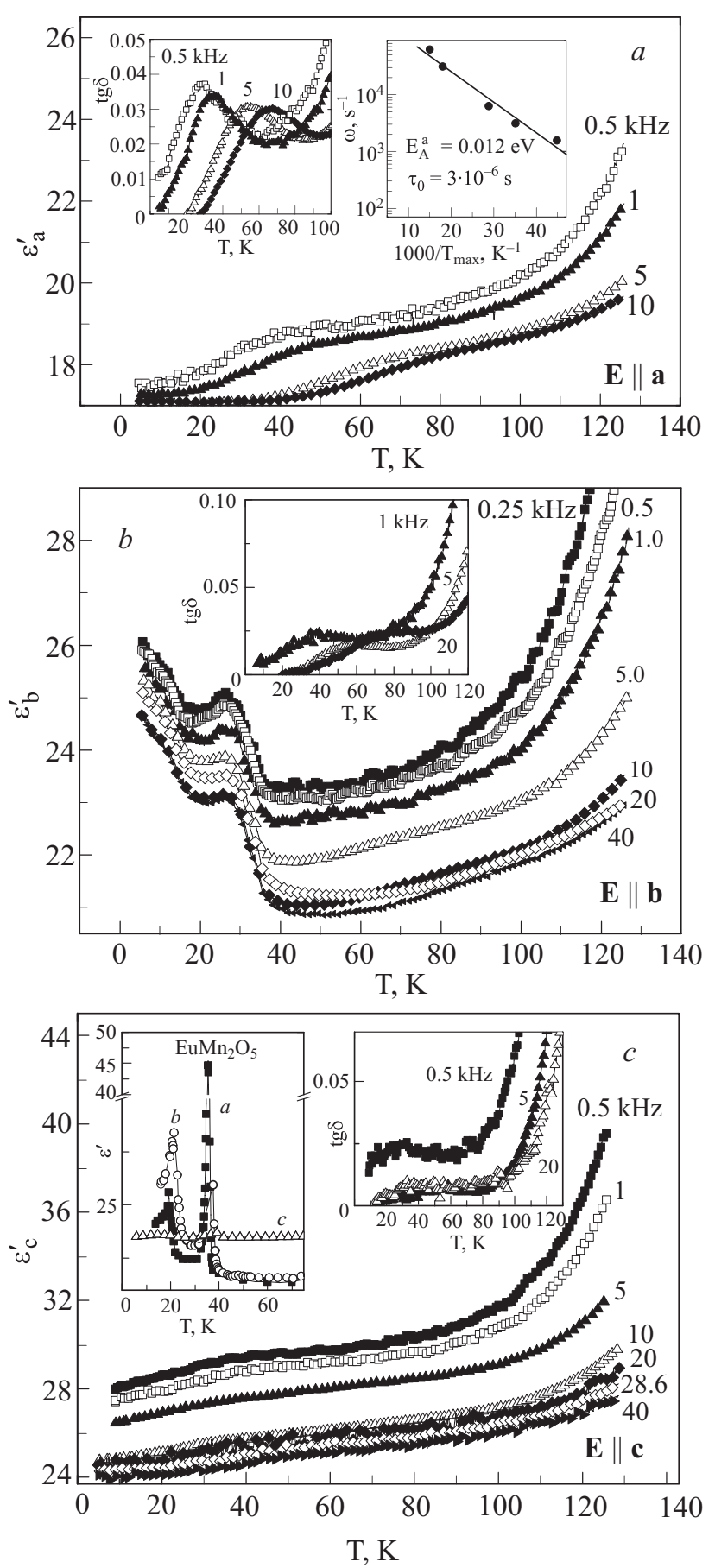

Fig. 2. Temperature dependences of the real part of permittivity $\varepsilon^{\prime}$ for ECMO for a number of frequencies $(\mathrm{kHz})$ indicated near the curves for different orientations of the ac electric field $\mathbf{E}: \mathbf{E}\|\mathbf{a}(a), \mathbf{E}\| \mathbf{b}(b)$, E $\|$ c $(c)$. Left inset to Fig. 2,a: temperature dependences of dissipation factor $\operatorname{tg} \delta$ for a number of frequencies $(\mathrm{kHz})$ indicated near the curves. Right inset to Fig. 2,a: Arrhenius law for the temperatures of dissipation factor maxima. Inset to Fig. 2,b: temperature dependences of $\operatorname{tg} \delta$ for a number of frequencies $(\mathrm{kHz})$ indicated near the curves. Left inset to Fig. 2,c: temperature dependences of $\varepsilon_{a, b, c}^{\prime}$ at $1 \mathrm{kHz}$ for EMO. Right inset to Fig. 2,c: temperature dependences of $\operatorname{tg} \delta$ for a number of frequencies $(\mathrm{kHz})$ indicated near the curves.
When analyzing dielectric properties of the doped crystals, we assume that the dielectric volume of the initial matrix and the 2D layers with charge carriers have the same magnetic and ferroelectric orderings. However, in 2D layers these orderings are frustrated by doping impurities and $\mathrm{Mn}^{3+}-\mathrm{Mn}^{4+}$ ion pairs. Dielectric and magnetic measurements and also neutron scattering data $[15,16]$ have shown that the sequence of phase transitions in EMO is as follows: magnetic phase transition with $T_{N} \approx 40 \mathrm{~K}$, ferroelectric phase transition with $T_{C} \approx 35 \mathrm{~K}$, and phase transition in the vicinity of $20 \mathrm{~K}$. Below $T_{N}$ magnetic ordering with wave vector $q=[1 / 2,0,0.3]$ occurs, i.e., there is an antiferromagnetic ordering along the $\mathbf{a}$ axis and incommensurate magnetic structure along the $\mathbf{c}$ axis. The ferroelectric phase transition in EMO arises somewhat below $T_{N}$. The polarization vector is oriented along the $\mathbf{b}$ axis. In the vicinity of the ferroelectric transition temperature the magnetic structure wave vector changes in a jump. At lower temperatures the wave vector $q=$ $=[1 / 2,0,1 / 3]$, i.e., the magnetic structure becomes commensurate [16]. A lower-temperature phase transition near $20 \mathrm{~K}$ is simultaneously structural and magnetic.

The relaxation processes of ECMO manifest themselves in the most pronounced way at $\mathbf{E} \| \mathbf{a}$. These processes can be attributed to displacements of the charge carriers responsible for recharging of $\mathrm{Mn}^{3+}-\mathrm{Mn}^{4+}$ ion pairs inside the $2 \mathrm{D}$ layers. This results in a phase separation into conductive ferromagnetic and dielectric antiferromagnetic domains. The phase separation is caused by double exchange (exchange interaction between neighboring $\mathrm{Mn}^{3+}$ and $\mathrm{Mn}^{4+}$ ions through $e_{g}$ electrons) with energy

$$
E_{d e}=-J \cos (\psi / 2) \mathbf{S}_{1} \mathbf{S}_{2} .
$$

Here, $\psi$ is the angle between the directions of localized spins $\mathbf{S}_{1}$ and $\mathbf{S}_{2}$ of neighboring $\mathrm{Mn}^{3+}$ and $\mathrm{Mn}^{4+}$ ions, and $J$ is the exchange constant $(J \approx 300 \mathrm{meV})$. Such an exchange gives rise to a ferromagnetic $S_{1}$ and $S_{2}$ orientation and charge carrier accumulation in the ferromagnetic domains $[5,6]$. Thus, the relaxation processes inside the $2 \mathrm{D}$ superlattice layers at $\mathbf{E} \| \mathbf{a}$ are accompanied by phase separation. The maxima in $\operatorname{tg} \delta$ satisfy the condition $\omega \tau=1$ for conductive ferromagnetic domains of different sizes ( $\omega$ is the frequency and $\tau$ is the domain lifetime). The frequency dependence of the temperatures of $\operatorname{tg} \delta$ maxima obeys the Arrhenius law (see Fig. 2,a)

$$
\tau=\tau_{0} \exp \left(E_{A}^{\mathrm{rel}} / k T_{\max }\right),
$$

where $\tau_{0}$ and $E_{A}^{\text {rel }}$ are the lifetime and characteristic activation barrier, respectively. In our case $\tau_{0} \approx 10^{-6} \mathrm{~s}$ and $E_{A}^{\text {rel }} \approx 12 \mathrm{meV}$. The charge carrier displacements in the layers are hindered by the antiferromagnetic indirect exchange interaction leading to an antiferromagnetic orientation of manganese ion spins along the a axis (in this case $\cos (\psi / 2)=0$ in Eq. (1)). $E_{A}^{\text {rel }} \approx 12 \mathrm{meV}$ corresponds 
to the magnitude of the indirect antiferromagnetic exchange. We suppose that a high $\tau_{0}\left(\tau_{0} \approx 10^{-6} \mathrm{~s}\right)$ is due to a low probability of the conductive domain decay as a whole.

At $\mathbf{E} \| \mathbf{b}$, the distribution of the barriers hindering charge displacements in the layers is mainly due to ferroelectric ordering. Above $35 \mathrm{~K}$, the barriers are determined by the interfaces between the domains of ferroelectric correlations which are preserved to the temperatures well above $35 \mathrm{~K}$ [17].

At $\mathbf{E} \| \mathbf{c}, \varepsilon^{\prime}$ is determined by the charge carrier displacements between the layers and, hence, the barriers at their boundaries.

The frequency dependence of $\varepsilon^{\prime}$ for all crystal orientations in a wide temperature interval below $100 \mathrm{~K}$ is due to the presence of layers and conductive domains in them. The layer response depends on the layer size. As frequency grows, responses from thinner layers or smaller domains inside the layers are detected. The thinner the layer, the higher the activation barrier at its boundary and the higher the carrier concentration in it. Charge carriers screen polarization and reduce $\varepsilon^{\prime}$.

The lower inset to Fig. 3 shows the $\varepsilon^{\prime}$ anisotropy at low temperatures. It can be seen that the minimal $\varepsilon^{\prime}$ is observed along the $a$ axis. At $\mathbf{E} \| \mathbf{c}$ the magnitude of $\varepsilon^{\prime}$ is the highest.

Let us now consider the effect of magnetic field $(\mathbf{H} \| \mathbf{a}$, $H=6 \mathrm{~T}$ ) on relaxation in ECMO at low temperatures. It can be seen from Fig. 4 that the relaxation processes are preserved as the field is applied but anomalies in $\varepsilon^{\prime}$ and $\operatorname{tg} \delta$ shift towards higher temperatures. In addition, these anomalies are more diffuse and their magnitudes are lower than those at $H=0$ (see inset to Fig. 4). A characteristic activation barrier in the Arrhenius law of $12 \mathrm{meV}$ at $H=0$ becomes equal to $78 \mathrm{meV}$. As before, we attribute the observed low-temperature relaxation in magnetic field to

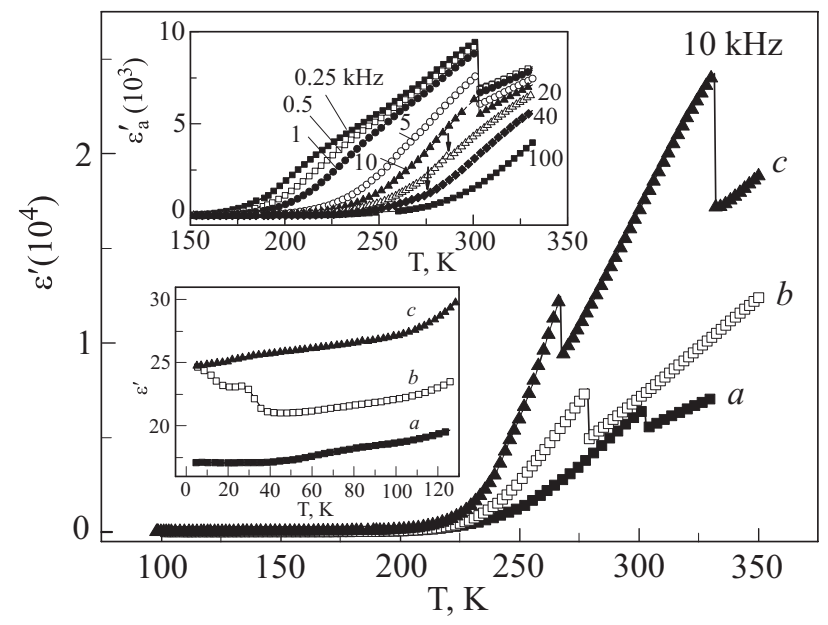

Fig. 3. Anisotropy of $\varepsilon_{a, b, c}^{\prime}$ for ECMO at $10 \mathrm{kHz}$. The lower inset: the same for low temperatures. The upper inset: temperature dependences of $\varepsilon_{a}^{\prime}$ at $\mathbf{E} \| \mathbf{a}$ for ECMO at some frequencies indicated near curves.

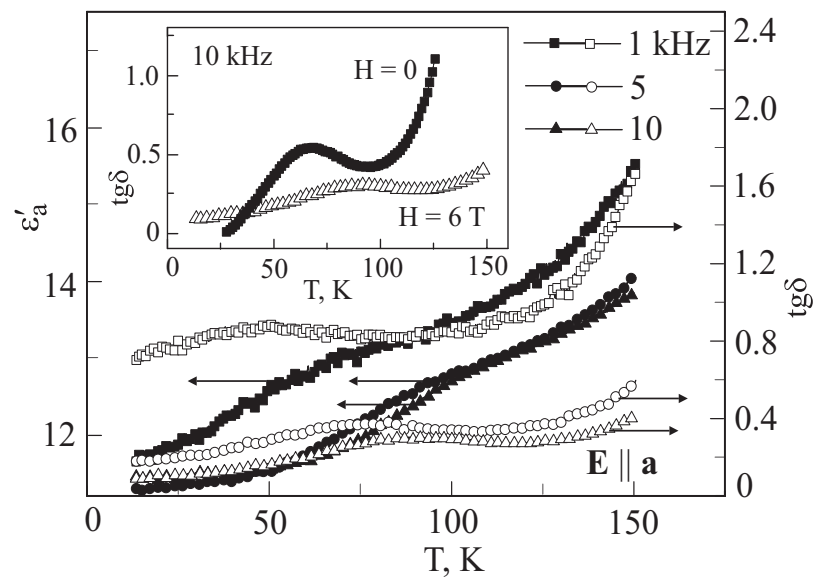

Fig. 4. Temperature dependences of $\varepsilon^{\prime}$ and $\operatorname{tg} \delta$ at $\mathbf{E} \| \mathbf{a}$ for ECMO for the several frequencies. Magnetic field $H=6 \mathrm{~T}, \mathbf{H} \| \mathbf{a}$, was applied at $5 \mathrm{~K}$ and the sample was heated under the applied field. The inset compares temperature dependences of tg $\delta$ at $\mathbf{E} \| \mathbf{a}$ in zero and nonzero magnetic field. Frequency is $10 \mathrm{kHz}$.

phase separation inside the quasi-2D layers. The magnetic field $\mathbf{H} \| \mathbf{a}$ increases the sizes of conductive ferromagnetic domains in the layers and enhances the barriers at the boundaries between these domains and dielectric antiferromagnetic domains due to double exchange.

\subsection{Magnetization processes}

Let us consider magnetization of the doped crystal along and transverse to the superlattice layers and compare it with magnetization of EMO. It is evident from Fig. 5 that the magnetization curves of ECMO radically differ from those of EMO.

The magnetization curves for EMO were found to be linear for the magnetic field orientations along all crystal axes (see the upper inset in Fig. 5,a) [15]. The magnetic moments along all axes in EMO are considerably lower than in ECMO. The magnetic moment along the a axis is the lowest, and that along the c axis is the highest.

It can be seen from Fig. 5 that the magnetic moments in the doped crystal are much higher than those in the initial crystal in all crystal directions. In spite of this fact the data for the doped crystal are fluctuating widely and more noisy than those for EMO. ECMO exhibits a strong magnetic anisotropy the character of which differs from that of the initial crystal. The magnetic moment along the a axis is much higher than that along the $\mathbf{c}$ axis. The magnetization curves are nonlinear and have jumps at some magnitudes of magnetic fields oriented both along the a and c axes. The magnetization behavior along the $\mathbf{c}$ axis changes irreversibly in the process of magnetic field cycling and the oscillations appear in the third cycle (Fig. 5,b). The oscillation period changes abruptly at the jump of the magnetization near $H=25$ kOe. This jump in magnetization one can relate to a magnetic phase transition. When the field decreases down to $H \sim 25 \mathrm{kOe}$ 

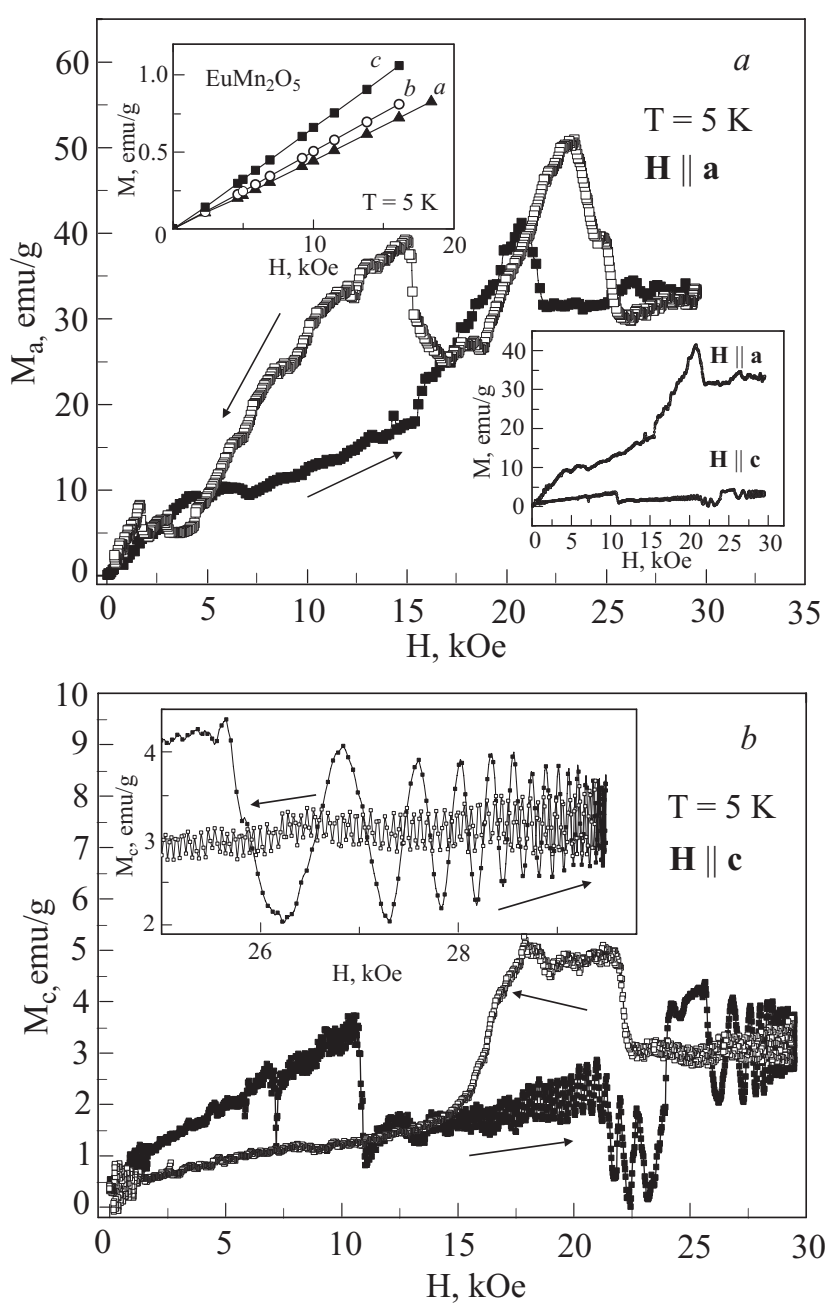

Fig. 5. Magnetic moment as a function of magnetic field for ECMO. $T=5 \mathrm{~K}$. $\mathbf{H}\|\mathbf{a}(a) . \mathbf{H}\| \mathbf{c}(b)$. The arrows show the directions of field variations. Upper inset in Fig. 5,a: magnetic moment as a function of magnetic field oriented along the principal axes of EMO. Lower inset in Fig. 5,a: anisotropy of magnetic moment with increasing field for ECMO. Inset in Fig. 5,b: oscillations of magnetic moment in high magnetic fields.

the oscillation period is constant and equal to the value observed at the highest field. This points to irreversible influence of the magnetic field on the properties of the crystal. We will show below that if the field stronger then $25 \mathrm{kOe}$ is applied at $T=5 \mathrm{~K}$ along the $\mathbf{c}$ axis, irreversibility manifest itself also in the dielectric properties of the crystal.

We believe that a considerable difference between magnetic properties of the doped and initial crystals is caused by the existence of superlattices. The magnetic system of ECMO consists of localized manganese ion spins and charge carrier spins providing recharging of $\mathrm{Mn}^{3+}-\mathrm{Mn}^{4+}$ ions in the 2D layers of superlattices. Localized manganese ion spins have a positive magnetic susceptibility, while the magnetic susceptibility of free charge carriers in the 2D layers can be negative. The negative susceptibility results from rotation of free charge carriers in the layers around the field applied normal to the layers, their orbital states being quantized in the magnetic field (Landau diamagnetism). Charge carriers can be free in the 2D layers if the spins of the $\mathrm{Mn}$ ions are ferromagnetically aligned in these layers.

Let us treat the magnetization curves of the doped crystal in more detail. As the magnetic field oriented along the a axis increases, a linear $M(H)$ dependence is observed under low fields $(H<5 \mathrm{kOe})$. In the field interval 5-17 kOe the magnetic moment decreases thus deviating from the linear $M(H)$ dependence. At $H>17 \mathrm{kOe}$ the moment begins to grow sharply again and exhibits a maximum at $H \approx 21 \mathrm{kOe}$. As the field further increases, the magnetic moment becomes saturated and fairly high, i.e., it exceeds by a factor of 40 the magnetic moment of EMO (in the same magnetic field). There is a hysteresis as the field decreases. The linear $M(H)$ dependence for ECMO (Fig. 5,a) at $(H<5$ kOe) results mainly from the predominant contribution of localized manganese ion spins. This is a weak longitudinal antiferromagnetic susceptibility of the dielectric antiferromagnetic phase. This contribution into the magnetic moment is the same for the initial and doped crystals. The major contribution into the magnetic moment of the doped crystal comes from a high positive ferromagnetic susceptibility of $\mathrm{Mn}^{3+}-\mathrm{Mn}^{4+}$ pairs in the 2D layers. As a result, the initial linear $M(H)$ slope for the doped crystals considerably exceeds that for the initial crystals. The ferromagnetic moment of $\mathrm{Mn}^{3+}-\mathrm{Mn}^{4+}$ pairs in the 2D layers grows with increasing magnetic field. The phase separation inside the layer leads to formation of ferromagnetic conductive domains. The higher the magnetic field the larger the domain. In this case the diamagnetic susceptibility caused by rotation of charge carriers normal to the magnetic field (across the layer) is weak. As a result, the total magnetic susceptibility (of localized spins and charge carriers) becomes nonlinear. The sharp jump in the magnetic moment at $H \approx$ $\approx 21 \mathrm{kOe}$ is related to the metamagnetic phase transition in the subsystem of localized manganese ion spins in the $2 \mathrm{D}$ layers. This transition occurs when the external magnetic field becomes equal to the internal effective antiferromagnetic field. The latter is weakened in the layers with ferromagnetic frustrations. After the metamagnetic transition a ferromagnetic ordering for all manganese ion spins is established in the layers. As the field further grows, the ferromagnetic moment becomes saturated, and its magnitude is rather high. Therefore, a high magnetic moment in the doped crystal at increasing magnetic field $\mathbf{H} \| \mathbf{a}$ is due to the ferromagnetism of localized manganese ion spins in the 2D layers, while the magnetic moment along the a axis of EMO is due to a low longitudinal antiferromagnetic susceptibility.

If $\mathbf{H} \| \mathbf{c}$, the magnetic field is oriented along the magnetic structure modulation vector. The modulated long-range magnetic order in the layers is also frustrated by ferromagnetic $\mathrm{Mn}^{3+}-\mathrm{Mn}^{4+}$ ion pairs. Application of a 
sufficiently high magnetic field along the $\mathbf{c}$ axis leads to formation of a homogeneous magnetic structure along this axis and, first of all, inside the layers. The magnetic moment jump at $H \approx 25 \mathrm{kOe}$ is observed. It is likely that a phase transition from the incommensurate phase into a homogeneous magnetic state of manganese ion spins in the layers occurs under this field (Fig. 5,b). Note that both the metamagnetic transition at $\mathbf{H} \| \mathbf{a}$ and transition at $\mathbf{H} \| \mathbf{c}$ are observed in nearly equal magnetic fields. This suggests that the latter transition is the spin-flip phase transition for antiferromagnetically ordered local spins in the layers. It could be expected that ferromagnetic moments in the layers due to local spin orientation would be nearly equal along the $a$ and $c$ axis, but in reality $M_{a} \gg M_{c}$ (Fig. 5). In all probability, this is due to a high diamagnetic susceptibility of free carriers in 2D layers at $\mathbf{H} \| \mathbf{c}$. As a result, the total magnetic moment along the $c$ axis is appreciably weakened, which leads to a strong magnetic anisotropy of the doped crystal.

The variation of magnetic field $\mathbf{H} \| \mathbf{c}$ in the course of cycling the field results in redistribution of the charge carrier concentrations between the 2D superlattice layers, thereby changing the barrier at the $2 \mathrm{D}$ layer boundaries and forming the dynamic equilibrium state of the superlattices.

The barrier at the 2D layer boundary can be written as

$$
W=W_{m}+W_{d e}-W_{C},
$$

where $W_{m}$ is the barrier in the lattice; $W_{d e}$ is the barrier due to the double exchange (Eq. (1)). The magnetic field, by leading to a ferromagnetic orientation of $S_{1}$ and $S_{2}$, increases the barrier at the layer boundary by $W_{d e}$. The Coulomb repulsion $W_{C}$ is [18]

$$
W_{C}=e^{2} /\left(\varepsilon_{0} R_{12}\right) \text {. }
$$

Here $e$ is the electron charge, $\varepsilon_{0}$ is the permittivity, and $R_{12}$ is the distance between charge carriers. As the concentration of charge carriers in the layers increases, the distances between them decreases, and the Coulomb repulsion that reduces the barrier becomes stronger [18]. As a result, a high enough magnetic field $\mathbf{H} \| \mathbf{c}$ form equilibrium 1D superlattices with a balance between attraction forces (double exchange, Jahn-Teller interaction) and Coulomb repulsion for charge carriers. The superlattices formed in the single crystal bulk of ECMO due to charge carrier selforganization in a magnetic field are similar to specially prepared semiconductor superlattices (see, e.g., [19]).

We believe that magnetic oscillations are excited in sufficiently high magnetic fields $\mathbf{H} \| \mathbf{c}$ in the dynamic equilibrium superlatticies and they are de Haas van Alphen (dHvA) oscillations [20]. It is known that the dHvA oscillation frequency $F$ for superlattices (in units of magnetic field) is [20]

$$
F=(\pi c \hbar / e) n=2.06 \cdot 10^{-7} n .
$$

It can be seen that $F$ is determined by the charge carrier concentration $n$ in the layer of the superlattice. Here, $c$ is the velocity of light and $\hbar$ is the Planck constant. In our case the oscillation frequency prior to the phase transition $F_{a}=10^{6}$ Oe, the charge carrier concentration being $n_{a}=$ $=5 \cdot 10^{12} \mathrm{~cm}^{-2}$. After the phase transition $F_{b}=10^{7} \mathrm{Oe}$ and $n_{b}=5 \cdot 10^{13} \mathrm{~cm}^{-2}$. Obtained values of the oscillation frequencies and charge carrier concentrations are typical for oscillations in semiconductor superlattices [20].

Note, that in our case the $\mathrm{dHvA}$ oscillations are excited in the internal field that is a sum of the field due to localized ferromagnetic moments of $\mathrm{Mn}$ and an external field. The oscillation period is determined by the internal field. Near the magnetic phase transition (at $H \approx 25 \mathrm{kOe}$ ) the oscillation period abruptly changes. Theoretically abruptly change of the frequency of $\mathrm{dHvA}$ oscillations near metamagnetic phase transition in antiferromagnetic metal is considered in [21]. The character of frequency change near the phase transition in our experiments is agreed with [21]. To estimate the charge carrier concentrations the frequency values taken sufficiently far from the phase transition were used.

Irreversibility of the state of the superlattices at decrease of the field we link with the lattice distortions due to the Jahn-Teller effect. They maintain higher barriers at the boundaries of the superlattices and a higher concentration of the charge carriers within them. It is the reason of oscillation period difference between the field going up and down cases.

Fluctuating and noisy character of the dependence of the magnetization on the external field is likely due to dynamic state realized in the superlattices. Continuous jumps of the charge carriers are typical for such state and they preserve a balance of the attractive and repulsive forces (the charge carrier self-organization). For these reasons the magnetic state of the superlattice layers is determined by self-organization.

\section{High temperatures}

\subsection{Dielectric properties}

As noted above, the room-temperature x-ray diffraction studies revealed the layered superstructure along the doped crystal $c$ axis. This means that noninteracting 1D superlattices assumed to be present at low-temperatures transform into a layered superstructure throughout the entire crystal volume with increasing temperature. As can be seen from Fig. 6, if $\mathbf{E} \| \mathbf{c}, \varepsilon^{\prime}$ and $\sigma_{a c}$ begin to grow above $150 \mathrm{~K}$. At $T \approx 185 \mathrm{~K}$ the $\varepsilon^{\prime}(T)$ and $\sigma_{a c}(T)$ dependences exhibit anomalies without frequency dispersion, which points to a phase transition at this temperature. Above $185 \mathrm{~K}$, both $\varepsilon^{\prime}$ and $\sigma_{a c}$ continue to grow and their frequency dispersion increases. The higher the frequency, the higher $\sigma_{a c}$ and the lower $\varepsilon^{\prime}$. 

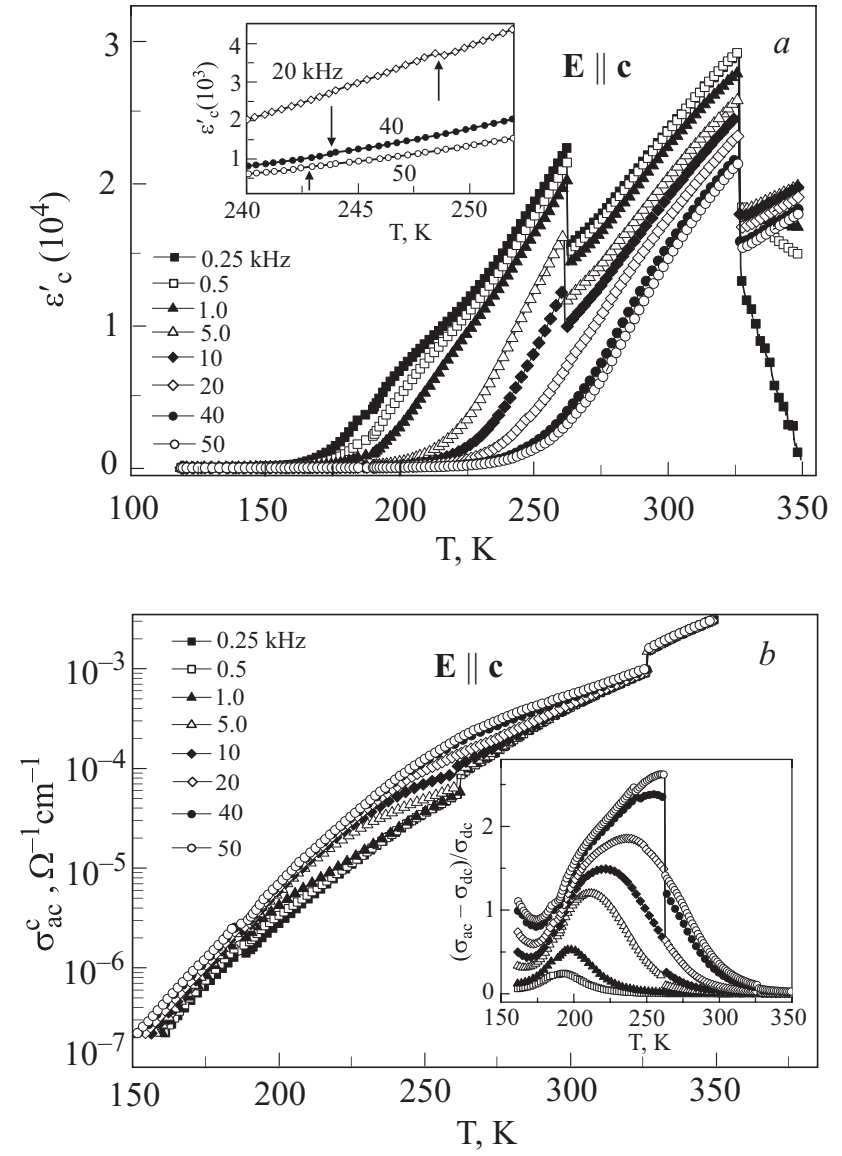

Fig. 6. Temperature dependences of $\varepsilon^{\prime}(a)$ and ac-conductivity $\sigma_{a c}(b)$ for ECMO for a number of frequencies $(\mathrm{kHz}), \mathbf{E} \| \mathbf{c}$. Inset to Fig. 6,a: anomalies on temperature dependences of $\varepsilon^{\prime}$ near $240 \mathrm{~K}$ for ECMO for a number of frequencies $(\mathrm{kHz})$, indicated near curves. Inset to Fig. 6,b: temperature dependences of local conductivity for a number of frequencies $(\mathrm{kHz})$.

The observed frequency dispersion of conductivity is typical of inhomogeneous systems containing restricted domains of different sizes of localized charge carriers with electron correlations [18]. The low-frequency $\sigma_{a c}$ and $\sigma_{d c}$ characterize percolation crystal conductivity that has no frequency dispersion. The $\sigma_{a c}$ with frequency dispersion characterizes local conductivities of small-size domains. These local conductivities are due to ac-losses caused by electron transfer between states deep within potential wells of the domains of electron correlations [18]. Temperature dependences of the local conductivities $\left(\sigma_{a c}-\sigma_{d c}\right) / \sigma_{d c}$ at different frequencies are shown in the inset to Fig. 6,b. They exist in ECMO in the temperature interval $160-330 \mathrm{~K}$. The frequency shifts of the temperature maxima of local conductivities are described by the Arrhenius law (Eq. (2)). The activation barrier for the high-frequency part of these conductivities is $E_{A}^{\sigma}=260 \mathrm{meV}$. The temperature interval in which the high-permittivity state exists coincides with that of the local conductivity. This means that $\varepsilon^{\prime}$ is mainly determined by the localized charge carriers. Of interest is the presence of jumps on the temperature dependences of $\varepsilon^{\prime}$ and $\sigma_{a c}$ near $260 \mathrm{~K}$ and at $T \approx 325 \mathrm{~K}$ (Fig. 6). Such jumps are observed with decreasing temperature as well and do not show hysteresis phenomena.

The first step-like anomalies in the $\varepsilon^{\prime}(T)$ dependences (near $260 \mathrm{~K}$ ) exhibit frequency dispersion. The higher the frequency the lower the jump temperature and the weaker the anomaly. The lower the frequency, the larger the size of the domain that gives response. At low frequencies there is almost no dispersion of the jumps in both $\varepsilon^{\prime}(T)$ and $\sigma_{a c}(T)$ and these jumps occur at $T \approx 262 \mathrm{~K}$. The dispersionfree anomalies at low frequencies can point to the phase transition at $T \approx 262 \mathrm{~K}$.

The $\varepsilon^{\prime}$ and $\sigma_{a c}$ jumps at $T \approx 325 \mathrm{~K}$ do not have frequency dispersion (Fig. 6). As temperature further grows, $\varepsilon^{\prime}$ drops to nearly zero at low frequencies. At high frequencies $\varepsilon^{\prime}$ continues to grow, probably to have more jumps at higher temperatures. The frequency dispersion of conductivity disappears, i.e., at $T \geq 325 \mathrm{~K}$ percolation conductivity dominates.

Figure 3 presents $\varepsilon^{\prime}$ anisotropy at all temperatures. It can be seen that $\varepsilon^{\prime}$ is the highest along the $\mathbf{c}$ axis and lowest along the a axis both at low and high temperatures. The $\varepsilon^{\prime}(T)$ dependences along the a axis and $\mathbf{b}$ axis have one jump each (at $T<350 \mathrm{~K}$ ), whereas two jumps are observed along the $\mathbf{c}$ axis. It is probable that more high-temperature jumps in $\varepsilon^{\prime}(T)$ along the a and $\mathbf{b}$ axis exist at $T>350 \mathrm{~K}$. Indeed, a frequency dispersion of the jumps in $\varepsilon^{\prime}(T)$ along the $\mathbf{a}$ axis near $300 \mathrm{~K}$ is similar to that of the anomalies near $260 \mathrm{~K}$ along the $\mathbf{c}$ axis (see the inset to Fig. 3).

Let us discuss how the state with a giant $\varepsilon^{\prime}$ is formed and what is the nature of the phase transitions observed. At E || c (Fig. 6), the hopping conductivity of charge carriers from the as-grown 2D layers increases and the number of carriers in the layers decreases with increasing temperature (at $T>100 \mathrm{~K}$ ). The electroneutrality is broken, and the layers become positively charged (owing to doping impurities $\mathrm{Ce}^{4+}$ ). Due to hops from the as-grown 2D layers, charge carriers (electrons) penetrate into the planes with manganese ions in the dielectric crystal matrix. In these planes recharging $\mathrm{Mn}^{4+}+e \leftrightarrow \mathrm{Mn}^{3+}$ takes place, and the layers become negatively charged. Coulomb attraction and annihilation of charged layers is inhibited by the barriers arising at their boundaries due to Jahn-Teller lattice distortions around $\mathrm{Mn}^{3+}$ ions. The probability of formation of negatively charged layers in the vicinity of all as-grown layers grows with increasing temperature. We suppose that the phase transition at $T \approx 185 \mathrm{~K}$ corresponds to a percolation phase transition, when an infinite cluster of charged layers alternating with dielectric interlayers of the initial crystal (a layered superstructure) is formed in ECMO.

The layered superstructure arising at $T \approx 185 \mathrm{~K}$ is inhomogeneous and consists of layers with different sizes, charge carrier concentrations, and barriers at their boundaries. This is the reason for appearance of the frequency dispersion in the $\varepsilon^{\prime}(T)$ and $\sigma_{a c}(T)$ dependences. Note that 
formation of the layered superstructure is accompanied by a change in the lattice state. A periodical charge distribution in the lattice gives rise to a state of the charge-ordering-induced ferroelectricity with the polarization along the $\mathbf{c}$ axis. Application of an ac field $\mathbf{E} \| \mathbf{c}$ leads to the barrier height modulation at the layer boundaries and charge displacement from one layer to another. In our opinion, this is the reason for a giant growth in permittivity above $185 \mathrm{~K}$.

The properties of the layered superstructure change above $185 \mathrm{~K}$ due to an increase in the probability of hops with increasing temperature. The barriers at the layer boundaries and charge carrier distribution between the superstructure layers at high temperatures are determined by the same interactions as in the 1D superlatticies at low temperatures in magnetic field (Eqs. (3), (4)), i.e., electronphonon, double exchange, and Coulomb interactions. The double exchange makes the barriers at the layer boundaries higher (attraction forces of charge carriers). However, filling of the layers is limited by Coulomb repulsion. As the charge carrier concentrations in the layers grow, the Coulomb repulsion responsible for barrier reduction increases. The balance between attraction and repulsion charge carrier interactions in the layers gives rise to an abrupt change in the charge carrier concentration.

At lower temperatures, thinner layers with the highest barriers at their boundaries have higher charge carrier concentrations. The thinner the layer, the higher the frequency of its response. As a result, jumps in $\varepsilon^{\prime}(T)$ occur at higher frequencies at lower temperatures (see the insets to Fig. 6,a and $6, b)$. As temperature grows, the charge carrier redistribution between the layers and generation of a more homogeneous stationary layered superstructure determined by the balance between attraction and repulsion charge carrier interactions takes place in the domains with larger sizes. This state is dynamic: there is a continuous carrier motion between local layers, which provides the balance between attraction and repulsion forces. The lower the frequency, the larger the domain in which this dynamic state occurs. At the lowest frequencies the dispersion is absent and the anomaly temperature becomes $T \approx 262 \mathrm{~K}$, i.e., the phase transition happens at this temperature. The phase transition at $262 \mathrm{~K}$ is attributable to formation to this dynamic homogeneous layered superstructure in the whole crystal. As temperature further grows, the superstructure decay and percolation conductivity growth start. We suppose that at $325 \mathrm{~K}$ the infinite cluster of the layered superstructure is destroyed. At $T>325 \mathrm{~K}$ localized carriers are preserved only in the smallsize isolated clusters formed from the layers with the highest barriers (see Fig. 6).

Let us turn now to the permittivity anisotropy. As evident from Fig. 3, a considerable growth in $\varepsilon^{\prime}$ along all principal crystal axes starts above $150 \mathrm{~K}$, and it is due to enhancement of hopping both inside and between the 2D layers. The anomalies in $\varepsilon^{\prime}$ and $\sigma_{a c}$ at $T \approx 185 \mathrm{~K}$ corresponding to the percolation phase transition are observed at all crystal orientations. Indeed, a layered superstructure can arise along one of the crystal axis (c axis) in an infinite crystal alone. In a finite-size sample, an edge layer structure along the $\mathbf{a}$ and $\mathbf{b}$ axes also arises. The $\varepsilon^{\prime}$ anisotropy in the samples is determined by the barrier anisotropy at the layer boundaries of the superstructure (see also Sec. 5.2). The barriers along the $\mathbf{c}$ axis are minimal; they are reduced by the internal electric field originnating from ferroelectricity along this axis. By analogy with the phase transition at $T=262 \mathrm{~K}$ (for the $\mathrm{c}$ axis), the $\varepsilon^{\prime}$ jumps at higher temperatures along the $\mathbf{a}$ and $\mathbf{b}$ axes can be attributed to the phase transition associated with the formation of an edge dynamic equilibrium superstructure.

\subsection{Dielectric spectra}

Figure 7 shows room-temperature dielectric spectra of ECMO for $\mathbf{E}$ oriented along principal crystal axes. The anisotropy observed is similar to that presented in Fig. 3 . There are abruptly falling $\varepsilon^{\prime}$ dependencies at definite frequencies typical of Debye relaxation. However, the $\varepsilon^{\prime \prime}$ maxima typically accompanying Debye relaxation jumps in $\varepsilon^{\prime}$ appear only slightly. This indicates that not only Debye relaxation is responsible for dielectric losses in ECMO. As a rule, such a behavior of $\varepsilon^{\prime \prime}$ points to a contribution of conductivity into dielectric losses.

As shown in Ref. 18, in semiconductors with random disorder and glasses with a wide range of restricted domains of variable scales with electron correlations, the contribution of conductivity of the domains into dielectric losses is described by Debye relaxation for all frequencies. On the other hand, the layered dielectrics containing layers with different conductivities and permittivities are characterized by dielectric losses described by Maxwell-Wagner relaxation. The dielectric spectra for the case of Maxwell-Wagner relaxation are [22, 23]

$$
\begin{gathered}
\varepsilon^{\prime}=\varepsilon_{\infty}+\frac{\varepsilon_{0}-\varepsilon_{\infty}}{1+\omega^{2} \tau^{2}}, \\
\varepsilon^{\prime \prime}=\frac{\sigma}{\omega}+\frac{\left(\varepsilon_{0}-\varepsilon_{\infty}\right) \omega \tau}{1+\omega^{2} \tau^{2}},
\end{gathered}
$$

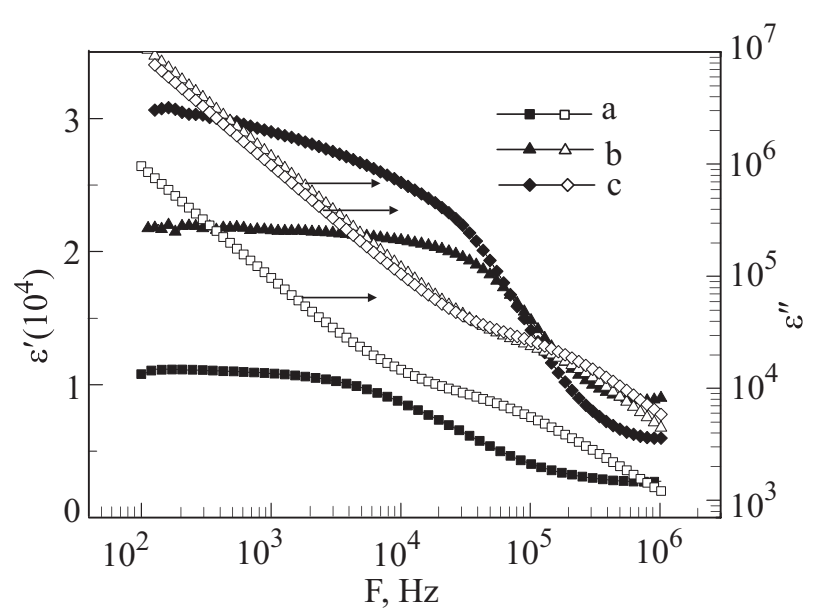

Fig. 7. Frequency dependences of the real $\varepsilon^{\prime}$ and imaginary $\varepsilon^{\prime \prime}$ parts of permittivity at room temperature for ECMO for $\mathbf{E} \| \mathbf{a}, \mathbf{b}, \mathbf{c}$. 
where $\sigma=1 / C_{0}\left(R_{1}+R_{2}\right)$ is the specific $\sigma_{a c} ; C_{0}$ is the geometrical factor of the sample; $R_{1}$ and $R_{2}$ are the layer resistances, and $\tau$ is the relaxation time for the layered sample. Equation (6) for $\varepsilon^{\prime}$ is the same for Debye and MaxwellWagner relaxations. The equation for the imaginary part of permittivity differs from that for the Debye relaxation by only an additional term associated with conductivity (the first term in Eq. (7)). It can be seen that this term is the highest at low frequencies, while the contribution of Debye relaxation at low frequencies is the lowest: $\varepsilon^{\prime \prime} \rightarrow 0$, when $\omega \rightarrow 0$.

Figure 8 presents dielectric spectra of ECMO at fixed temperatures for $\mathbf{E} \| \mathbf{c}$. The $\varepsilon^{\prime}$ spectra exhibit the behavior typical of Debye relaxation, $\varepsilon^{\prime}$ decreasing abruptly with temperature. These data are in good agreement with Fig. 6. Figure $8, b$ shows two curves, i.e., the summary $\varepsilon^{\prime \prime}$ and maxima in $\varepsilon^{\prime \prime}$ obtained by subtracting $\sigma$ (obtained from the slope of the linear dependence of summary $\varepsilon^{\prime \prime}$ on inverse frequency) from the total $\varepsilon^{\prime \prime}$. In this case the $\varepsilon^{\prime \prime}$ maxima usual of Debye relaxation manifest themselves. The frequency shifts of the temperatures of $\varepsilon^{\prime \prime}$ maxima are well described by Arrhenius relation (2). The magnitudes of $E_{A}$ are $260-276 \mathrm{meV}$, depending on the $\mathbf{E}$ direction. The
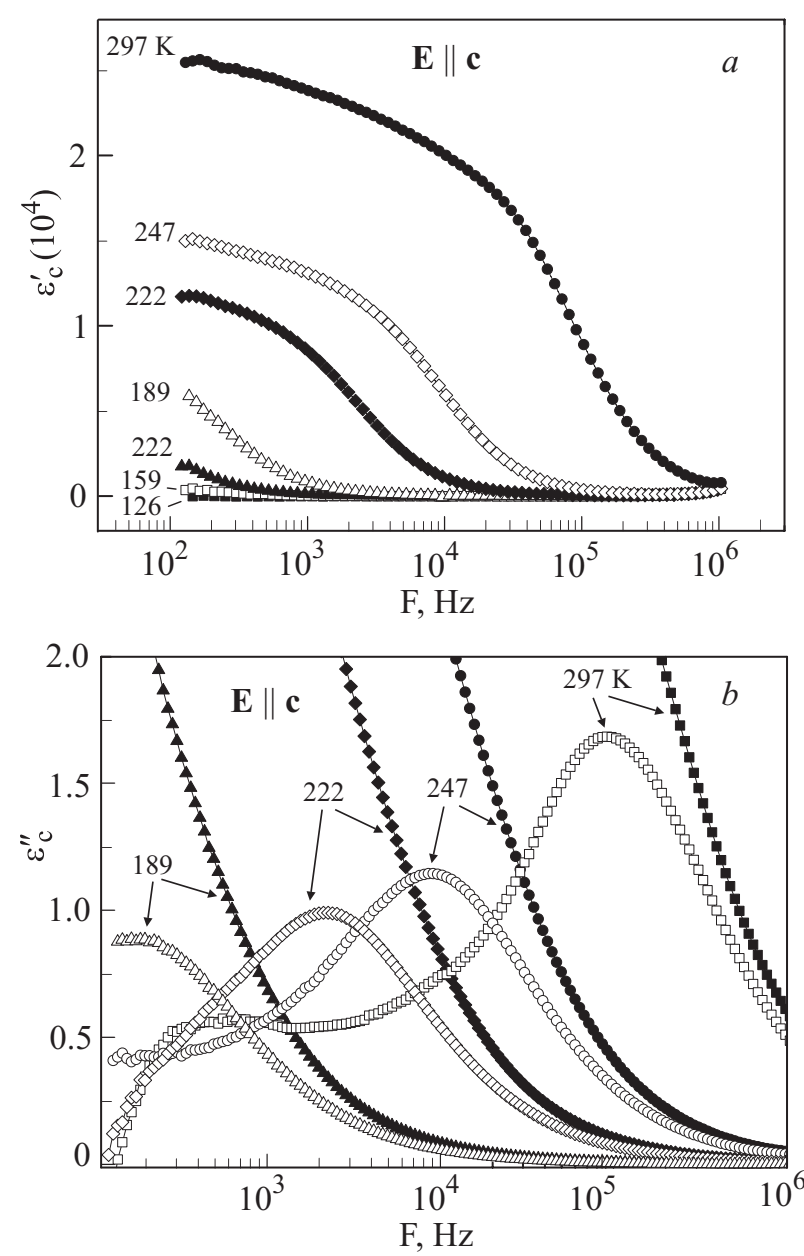

Fig. 8. Frequency dependences of $\varepsilon^{\prime}(a)$ and $\varepsilon^{\prime \prime}(b)$ for ECMO for a number of temperatures $(\mathrm{K})$ indicated near the curves. $\mathbf{E} \| \mathbf{c}$. highest $E_{A}$ is observed for $\mathbf{E} \| \mathbf{a}$, and the lowest $E_{A}$ is for $\mathbf{E} \| \mathbf{c}$. Note that frequency shifts of temperature maxima of localized conductivity in Fig. 6,b for $\mathbf{E} \| \mathbf{c}$ gave the same activation barrier of $260 \mathrm{meV}$.

Thus, the high-frequency part of localized conductivity is determined by Debye relaxation, and the low-frequency part is determined by Maxwell-Wagner relaxation. In our case the low-frequency part of $\sigma_{a c}$ and $\sigma_{d c}$ give contributions into the internal Maxwell-Wagner relaxation caused by the presence of a layered superstructure in the crystal bulk.

\subsection{Effect of magnetic field on dielectric properties}

Now we consider the effect of magnetic field on the formation of the layered superstructure state in ECMO. The magnetic field which is high enough to form the equilibrium $1 \mathrm{D}$ superlattices is applied at $T=5 \mathrm{~K}$ along the $\mathbf{c}$ and $\mathbf{a}$ axes.

As can be seen from Fig. 9, application of $\mathbf{H} \| \mathbf{c}, H \geq$ $\geq 25 \mathrm{kOe}$ at a low temperature increases the temperature of the highly polarized state formation from 185 to $225 \mathrm{~K}$. In this case $\varepsilon^{\prime}$ grows more abruptly than for $H=0$. Application of a lower magnetic field does not produce any effect.

There is a considerable hysteresis for formation of the highly polarized state at sample heating and cooling in a magnetic field. The cooling curve nearly coincides with the initial (prior to field application) curve. Note that no temperature hysteresis is observed for formation of this state in zero magnetic field (see the inset to Fig. 9).

The state with a giant permittivity arising in ECMO at $T>225 \mathrm{~K}$ has a different barrier distribution in the lattice as compared with the state at $H=0$. A characteristic activation barrier in the Arrhenius law is $E_{A} \approx 550 \mathrm{meV}$

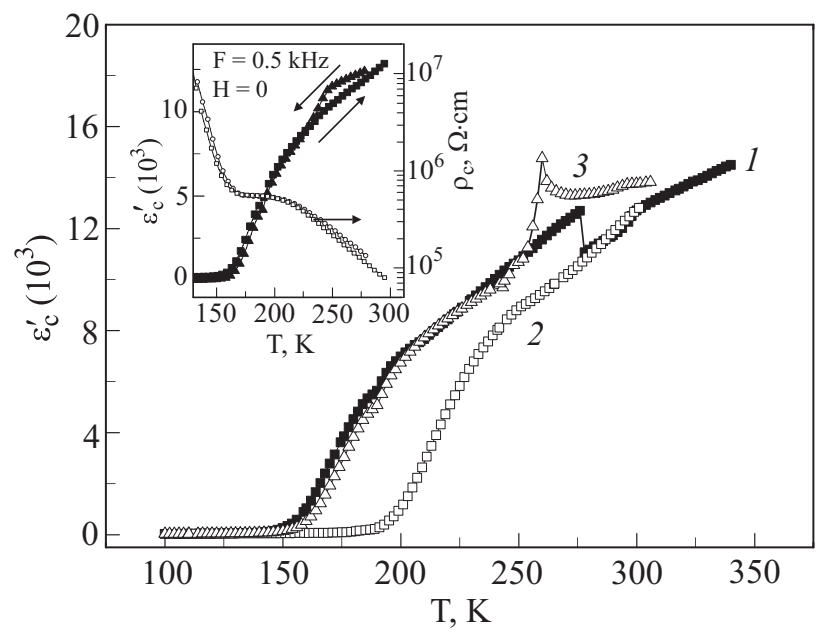

Fig. 9. Temperature dependences of $\varepsilon^{\prime}, \mathbf{E} \| \mathbf{c}$, for the initial crystal state of ECMO in zero magnetic field (curve 1, cooling). Curve 2 is sample heating in magnetic field $H=6 \mathrm{~T}(\mathbf{H} \| \mathbf{c})$ applied at $T=5 \mathrm{~K}$. Curve 3 is sample cooling (ZFC) after the field is removed at the highest temperature. Frequency is $0.5 \mathrm{kHz}$. Inset: temperature hysteresis for ECMO prior to magnetic field application. 
(instead of $E_{A} \approx 260 \mathrm{meV}$ at $H=0$ ). This change in the barrier is comparable to the magnitude of double exchange $(J \sim 300 \mathrm{meV})$.

The layered superstructure in the doped crystal formed at high temperatures after application of a high magnetic field does not decay when the magnetic field is removed. When the field is removed at a high temperature and the sample is cooled in zero field (ZFC), the $\sigma$ and $\varepsilon^{\prime}$ curves, as noted above, are similar to the cooling curves prior to field application. However, when the sample is again heated in zero magnetic field (ZFH), $\sigma$ and $\varepsilon^{\prime}$ behave as for the heating regime in the magnetic field. Therefore, the metastable but long-living state arises in ECMO after application of a sufficiently high magnetic field at a low temperature. A slow relaxation (during 15 days) to the initial state (prior to magnetic field application) occurred in our experiments.

\section{Conclusion}

Single crystals of diluted ferroelectromagnetic-semiconductors ECMO that exhibit a giant permittivity $\left(\varepsilon^{\prime} \sim 10^{4}\right)$ above $185 \mathrm{~K}$ have been synthesized and studied. The experimental data obtained in our investigations can be adequately explained if we assume that the as-grown quasi2D layers that contain doping impurities, charge carriers (electrons), and manganese ions of variable valence $\mathrm{Mn}^{3+}-$ $\mathrm{Mn}^{4+}$ are formed in ECMO normal to the $\mathbf{c}$ axis (1D superlattices). At low temperatures the superlattices are electrically neutral and occupy a small crystal volume. The major crystal volume is occupied by the dielectric matrix of the initial EMO crystal. At $T=5 \mathrm{~K}$ in magnetic filed $\mathbf{H} \| \mathbf{c}$ the $\mathrm{dHvA}$ oscillations were observed that gives evidence for the existing of superlattices.

As temperature grows and, hence, the probability of thermally activated charge carrier hops from the layers increases, a layered superstructure consisting of charged layers alternating with dielectric interlayers of the initial crystal is formed. This is a dynamic state with a periodical charge distribution accompanied by lattice distortions. It can be classified as ferroelectricity due to charge ordering leading to a giant permittivity. The ferroelectricity state arises due to a percolation phase transition at $T \approx 185 \mathrm{~K}$ and exists up to $325 \mathrm{~K}$. The crystal electroneutrality in this temperature interval is provided by the equality between the number of excess doping electrons and difference between the $\mathrm{Mn}^{3+}$ and $\mathrm{Mn}^{4+}$ concentrations.

Double exchange between $\mathrm{Mn}^{3+}$ and $\mathrm{Mn}^{4+}$ ions that gives rise to a ferromagnetic orientation of spins of these ions plays an important role in ECMO at all temperatures. Together with the Jahn-Teller and Coulomb interactions, it forms barriers at the superlattices and the superstructure layer boundaries.

The presence of the layered superstructure has been confirmed in x-ray diffraction studies of ECMO. Thus, it has been found that ECMO is really a ferroelectromagnetic semiconductor with high temperatures (above $185 \mathrm{~K}$, including room temperature) of magnetic and ferroelectric orderings. A strong coupling between magnetic and polar states in these crystals is achieved due to the participation of charge carriers in formation of both the magnetic and ferroelectric long-range orders.

The authors would like to thank N.V. Zaitzeva for X-ray phase analysis of the samples.

The work was supported by the Russian Foundation for Basic Research (grant 08-02-00077) and Presidium of RAS (Programme 03).

1. Y. Noda, H. Kimura, M. Fukunaga, S. Kobayashi, I. Kagomiya, and K. Kohn, J. Phys.: Condens. Matter 20, 434206 (2008).

2. P.G. Radaelli and L.C. Chapon, J. Phys.: Condens. Matter 20, 434213 (2008).

3. A.B. Harris, M. Kenzelmann, A. Aharony, and O. EntinWohlman, Phys. Rev. B78, 014407 (2008).

4. A.M. Kadomtseva, S.S. Krotov, Yu.F. Popov, and G.P. Vorob'ev, Fiz. Nizk. Temp. 32, 933 (2006) [Low Temp. Phys. 32, 709 (2006)].

5. L.P. Gor'kov, Phys. Usp. 41, 589 (1998).

6. M.Yu. Kagan and K.I. Kugel, Phys. Usp. 44, 553 (2001).

7. J. van den Brink and D.I. Khomskii, J. Phys.: Condens. Matter 20, 434217 (2008).

8. E.I. Golovenchits and V.A. Sanina, JETP Lett. 81, 509 (2005).

9. E.I. Golovenchits and V.A. Sanina, JETP Lett. 84, 190 (2006).

10. V.A. Sanina, E.I. Golovenchits, and V.G. Zalesskii, Phys. Solid State 50, 913 (2008).

11. V.A. Sanina, E.I. Golovenchits, and V.G. Zalesskii, Phys. Solid State 50, 922 (2008).

12. V.A. Sanina, L.M. Sapozhnikova, E.I. Golovenchits, and N.V. Morozov, Sov. Phys. Solid State 30, 1736 (1988).

13. R.D. Shannon, Acta Crystall. A32, 751 (1976).

14. Z.H. Sun, B.L. Cheng, S. Dai, K.J. Jin, Y.L. Zhou, Y.B. Lu, Z.H. Chen, and G.Z. Yang, J. Appl. Phys. 99, 084105 (2006).

15. E.I. Golovenchits, N.V. Morozov, V.A. Sanina, and L.M. Sapozhnikova, Sov. Phys. Solid State 34, 56 (1992).

16. V. Polyakov, V. Plakhty, M. Bonnet, P. Burlet, L.P. Regnault, S. Gavrilov, I. Zobkalo, and O. Smirnov, Physica B297, 208 (2001).

17. E.I. Golovenchits, V.A. Sanina, and A.V. Babinskii, JETP 85, 156 (1997).

18. A.R. Long, Adv. Phys. 31, 587 (1982).

19. Semiconductor Superlattices. Growth and Electronic Properties, H.T. Granhm (ed.), World Scientific, Singapore (1995).

20. D. Shoenberg, Magnetic Oscillations in Metals, Cambridge University Press (1984).

21. A.D. Balaev, V.V. Val'kov, V.A. Gavrichkov, N.B. Ivanova, S.G. Ovchinnikov, and V.K. Chernov, Uspehi Fiz. Nauk 167, 1016 (1997).

22. A.R. von Hippel, Dielectrics and Waves, N.Y., London (1954).

23. D. O'Neill, R.M. Bowman, and J.M. Gregg, Appl. Phys. Lett. 77, 1520 (2000). 